\title{
Erratum to: An Analysis on Decentralized Adaptive MAC Protocols for Cognitive Radio Networks
}

\author{
Munam Ali Shah Sijing Zhang Carsten Maple
}

Erratum to: International Journal of Automation and Computing DOI: 10.1007/s11633-013-0695-z

The original version of this article unfortunately contained a mistake. The received date and revised date were incorrect. The corrected received date and revised date are given below.

Manuscript received October 2, 2012; revised November 1, 2012

The online version of the original article can be found at http://dx.doi.org/10.1007/s11633-013-0695-z. 